\title{
Une expérience de voyage éco et ethno- touristique chez les Wayuu. Témoignage et analyse
}

\section{Karen López Hernández}

\section{(2) OpenEdition \\ Journals}

Édition électronique

URL : http://journals.openedition.org/elohi/467

DOI : $10.4000 /$ elohi.467

ISSN : 2268-5243

Éditeur

Presses universitaires de Bordeaux

\section{Édition imprimée}

Date de publication : 1 janvier 2015

Pagination : 49-73

ISBN : 979-10-300-0044-3

ISSN : 2431-8175

Référence électronique

Karen López Hernández, « Une expérience de voyage éco et ethno-touristique chez les Wayuu.

Témoignage et analyse », ELOHI [En ligne], 7 | 2015, mis en ligne le 01 janvier 2016, consulté le 01 mai 2019. URL : http://journals.openedition.org/elohi/467 ; DOI : 10.4000/elohi.467 


\section{Une expérience de voyage éco et ethno-touristique chez les Wayuu. Témoignage et analyse}

\section{KAREN LÓPEZ HERNÁNDEZ}

ORACLE (Observatoire Réunionnais des Arts, des Civilisations et

des Littératures dans leur Environnement), Université de La Réunion

\section{Introduction}

Les Wayuu habitent dans la péninsule de La Guajira, au nord de la Colombie et à l'ouest du Venezuela, en Amérique du Sud. La température de cette région oscille, en moyenne, entre 35 et 40 degrés (Informe socioeconómico del Departamento de La Guajira 2014 : 91). À cause de ses conditions climatiques et topographiques, cette région a été considérée comme un territoire désertique, vide, stérile, hostile. Cependant, La Guajira présente une variété écologique différenciée en trois régions naturelles : Haute, Moyenne et Basse. La plupart des Wayuu habitent dans la Moyenne et Haute Guajira. Cette dernière est la zone la plus sèche et la pénurie d'eau est une des caractéristiques de l'environnement (Meisel : 6, 9 et 48). Notre histoire se déroule dans la Haute Guajira.

Les Wayuu sont organisés en clans matrilinéaires et, selon Benson Saler (30), ils se caractérisent par le fait de ne pas avoir de liens d'obligations sociales, politiques et économiques entre eux. L'élevage, des caprins et des ovins principalement, est l'activité la plus importante dans la mesure où ils pensent que la richesse est fonction du nombre d'animaux qu'ils possèdent. Pour Michel Perrin, « la taille des troupeaux est, avec la possession de certains bijoux, la plus grande marque de prestige » $(2001: 25)$. Très hiérarchisée, la société wayuu a maintenu un contact vif avec la société guajira créole colombienne (Perrin, 2001:25). $\mathrm{Du}$ fait de cette forte relation, les Wayuu font un "va-et-vient » entre les rancherías $^{1}$ et les villes. D’après le recensement de 2005 mené par le Département

1. Lieu de résidence wayuu. 
de Statistiques Colombien (DANE), la population ajustée du département de La Guajira colombienne est de 681575 personnes, dont plusieurs groupes de Primo-natifs (Wayuu, Kogui, Wiwa, Arhuaco) qui représentent $44,9 \%$ de la population totale ${ }^{2}$. Les Wayuu en constituent $38 \%^{3}$ et leur langue, le wayuunaiki, de la famille arawak, est une langue officielle régionale.

La Guajira a été historiquement ignorée par les centres de pouvoir politique et économique de la Colombie (Orsini : 33). Pendant l'époque de la Conquête, cette région était difficile à contrôler pour les conquérants, autant du fait de conditions environnementales comme le manque d'eau (Polo Acuña 1998 : 3334) que de la résistance des Wayuu (Polo Acuña, 2012: 79-80). Mais, avec la mondialisation et la découverte de ressources inestimables (pétrole, charbon, gaz, énergie éolienne et solaire), les enjeux de La Guajira ont changé ; à la fin des années 1970, ce département est devenu un territoire prometteur pour le développement (Hutin : 227). Nous pouvons considérer que l'idée de désertsource supplante soudain celle de désert-vide. La région est censée s'incorporer à la dynamique du développement du pays.

Grâce aux atouts naturels et culturels, l'écotourisme et l'ethno-tourisme s'ouvrent comme des activités économiques qui promettent un développement à La Guajira colombienne (Meisel : 48). Le Plan de Desarrollo ${ }^{4}$ de ce département de la Colombie leur donne une importance majeure pour le progrès de la région : "Nous voudrions positionner La Guajira comme une destination touristique nationale et internationale " (Plan de Desarrollo : 59). Bien que l'infrastructure touristique soit limitée, des touristes visitent ce département, plus particulièrement le Cabo de la Vela ${ }^{5}$, un des endroits les plus promus (Meisel, : 48-49). En 2012-2013, 0,3\% des Colombiens ont visité La Guajira (Informe socioeconómico del Departamento de La Guajira 2013 : 78). La Direction du Département du Tourisme a estimé à 200 touristes étrangers et 3000 nationaux les visiteurs de La Guajira colombienne pendant la Semaine sainte, en $2014^{6}$.

2. Departamento Administrativo Nacional de Estadísticas, Colombia, site Dane [en ligne], page consulté le 6 juin 2014. Disponibilité et accès : https://www.dane.gov.co/ files/censo2005/etnia/sys/colombia_nacion.pdf.

3. Sistema Nacional de Información Cultural, Colombia, site SINIC [en ligne], page consulté le 6 juin 2014. Accès : http://www.sinic.gov.co/SINIC/ColombiaCultural/ColCulturalBusca.aspx?AREID=3\&SECID=8\&IdDep=44\&COLTEM=216.

4. Document exposant la politique de planification de chaque département pour une période gouvernementale.

5. Grâce à ses plages, le Cabo de la Vela, le cap, est un des lieux les plus réputés de La Guajira.

6. D'après un article du Diario del Norte [en ligne], page consulté le 4 mars 2014. Disponibilité et accès http://diariodelnorte.net/noticias/generales/11242-temporada-turistica-supero-las-expectativas-en-la-guajira.html. 
Les Primo-natifs ${ }^{7}$ wayuu se sont intégrés à cette dynamique économique. Nous avons pu constater que des familles wayuu offrent leurs logements et proposent des activités récréatives aux touristes avec la mise en spectacle de certaines traditions. Ce lien nouveau est l'objet de notre recherche.

Le tourisme est un objet d'étude qui peut être saisi à partir des analyses des interactions et des situations que mettent en œuvre les représentations des différents acteurs. Pour cela, le concept d'imaginaire dialogique peut apporter des éclairages sur la compréhension des interactions dans un voyage touristique. Selon Marie-Thérèse Vasseur :

Cet imaginaire dialogique nous renvoie aux différentes dimensions du dialogue. Il englobe en effet :

- l'image que chaque locuteur se fait de l'autre,

- l'image qu'il se fait de la tâche,

- l'image qu'il se fait de la situation,

- enfin l'image qu'il se fait de ce que l'on peut faire avec le langage, par exemple, obtenir quelque chose que l'on désire, paraître intelligent, aider l'autre... (Vasseur : 106)

L'imaginaire dialogique constitué par les échanges tripartites (concepteurs des voyages-touristes-populations natives) met en jeu les capitaux culturels, historiques, écologiques ou ethnologiques dans une course à la mise en exergue de la différence. Nous pouvons considérer que ces capitaux sont en effet, pour les concepteurs, autant d'atouts qui permettent aux utilisateurs - touristes et populations natives - de faire la différence entre les propositions résultant de leurs combinaisons. S'agissant, comme sur tout marché, de faire beaucoup mieux que les autres, il est raisonnable de penser que l'ambition de cultiver la différence devient, sur le marché touristique, un enjeu redoutable pour les cultures locales. Cette logique s'explique aussi par le concept de "marketing territorial » travaillé par Elizabeth Cunin et Christian Rinaudo :

la mise en scène de l' "authenticité " des traditions locales ou du patrimoine immatériel s'inscrit également dans une logique de marketing territorial, qui propose un espace touristique dans lequel la diversité des populations et la différence culturelle sont des arguments d'attractivité et des ressources locales pouvant être déclinées en articles de consommation (artisanat "ethnique", productions culturelles, visites de villages, festivals de musiques ou de danses traditionnelles, parcs d'attractions...) (Cunin et Rinaudo : 140).

Ainsi, la mise en valeur des villes, des forêts, des déserts, de toute sorte de sites invite au dépaysement recherché par des touristes. Associées souvent à ces sites,

7. Nous employons ce terme pour échapper à la charge idéologique des mots tels qu'indigène, indien ou amérindien. Il fait allusion aux populations habitant cette région avant l'arrivée des Européens. 
les populations locales et leurs cultures sont valorisées par un «marketing territorial », ce qui est déjà une invitation à les connaître dans les limites du voyage... Pourtant, certaines offres vont plus loin en proposant, par exemple, des «voyages initiatiques » comme les expériences chamaniques : c'est le cas de la ville d'Iquitos, au Pérou, devenue «La Mecque de l'ayahuasca » (Losonczy et Mesturini : 94, 96) ${ }^{8}$.

C'est dans cette perspective que nous voulons situer notre analyse, en prenant l'exemple d'un voyage éco et ethno-touristique. Quelles peuvent être les représentations de l'altérité dans un face-à-face médiatisé par le marché touristique ? Comment les places dialogiques permutent-elles et font-elles émerger des objectifs et des rôles inattendus dans les relations marchandes établies par le tourisme? Pour ce faire, nous allons analyser un cas où nous étions impliquées comme guide et ethnologue. La relation triangulaire (touristes, guide natif et nous) a eu des retombées négatives et les trois acteurs ont fini le périple dans un état de déception. En outre, "les malentendus " ${ }^{9}$, dus, en partie, à la divergence des représentations où les uns et les autres ne se retrouvent pas, ont été au cœur de ce voyage. Dans un premier temps, nous allons exposer brièvement l'offre touristique de la population wayuu pour situer les conditions matérielles, un aspect d'une grande importance pour les touristes. Nous allons, ensuite, analyser les représentations des touristes et le rôle du guide natif à partir du parcours touristique réalisé avec nous, sachant que nous avons partagé le rôle de guide. Finalement, nous nous demanderons quelles sont les contributions économiques provenant de l'écotourisme pour les familles natives qui participent à cette activité. L'histoire a eu lieu en janvier 2009, dans La Guajira colombienne, chez les Wayuu ${ }^{10}$.

8. La consommation de l'ayahuasca, breuvage végétal, est une pratique ancienne des peuples premiers de la région amazonienne. Consommée lors d'un rituel encadré par des spécialistes dans le chamanisme, «l'ayahuasca a acquis la réputation d'être un hallucinogène puissant, induisant des visions à caractère mystique » (Losonczy et Mesturini : 94). Des voyages organisés sont mis en œuvre dans plusieurs sites de cette région, dont Iquitos. Pour les chercheures Losonczy et Mesturini $(94,96)$, « la courte durée du 'voyage touristique organisé' n'épuise pas l'ampleur du phénomène qui inclut aussi des voyages-quête à multiples étapes et de longs séjours d'apprentissage... ».

9. Dans sa recherche sur le tourisme solidaire dans une ville burkinabé, Nadège Chabloz analyse ce phénomène qu'est le malentendu, qu'elle propose de conceptualiser et de décliner en fonction des faits et des conséquences à ces faits qu'elle a pu observer (Chabloz : 34 et suivantes).

10. Quoique l'histoire à analyser se déroule en 5 jours, début janvier 2009, il convient de préciser que nous sommes originaire de La Guajira et avons réalisé un travail de terrain dans cet espace géographique de plusieurs mois, entre les années 2004 et 2012. 


\section{L'offre éco et ethno-touristique dans la Haute Guajira}

Nous allons évoquer l'image sur laquelle certaines agences touristiques fondent leur publicité du lieu où se déroule notre histoire, la Haute Guajira, puis décrire trois offres touristiques des Wayuu. Au préalable, nous décrirons le cadre institutionnel dans lequel ces initiatives ont pris corps.

Dès qu'on arrive au Cabo de la Vela, Haute Guajira, on aperçoit de petits kiosques, ou bungalows, qui offrent des jus, des boissons alcoolisées, de l'eau ; on entend de la musique et de petites lumières électriques s'allument le soir, au bord de la plage. Ces images contrastent avec celles, plus anciennes, où le Cabo était réputé pour son silence et son isolement. En effet, le Cabo de la Vela a changé, plus particulièrement sous le gouvernement de Álvaro Uribe ${ }^{11}$, qui a promu l'écotourisme en Colombie.

\section{Cadre politique colombien de l'écotourisme communautaire}

En 1996 le gouvernement colombien a formulé la Loi Générale du Tourisme, Loi $300^{12}$. Conçu comme une industrie avec un potentiel économique important pour le développement durable, le tourisme est réglementé et contrôlé par l'État ; les communautés et le secteur privé sont censés participer. Cette loi définit plusieurs formes de tourismes : écotourisme, ethno-tourisme, tourisme métropolitain. Selon la Loi :

El Ecoturismo es aquella forma de turismo especializado y dirigido que se desarrolla en áreas con un atractivo natural especial y se enmarca dentro de los parámetros del desarrollo humano sostenible. El Ecoturismo busca la recreación, el esparcimiento y la educación del visitante a través de la observación, el estudio de los valores naturales y de los aspectos culturales relacionados con ellos. Por lo tanto, el Ecoturismo es una actividad controlada y dirigida que produce un mínimo impacto sobre los ecosistemas naturales, respeta el patrimonio cultural, educa y sensibiliza a los actores involucrados acerca de la importancia de conservar la naturaleza. El desarrollo de las actividades ecoturísticas debe generar ingresos destinados al apoyo y fomento de la conservación de las áreas naturales en las que se realiza y a las comunidades aledañas (Artículo 26, Ley 300 de 1996).

En 2006, le gouvernement de Álvaro Uribe définit des axes, "Lineamientos para una política de ecoturismo comunitario en Colombia $»^{13}$. L'objectif : rendre plus autonomes les communautés dans la gestion et le contrôle des bénéfices. Pour ce faire, les axes cherchent à structurer les bases pour orienter les

11. Álvaro Uribe, Président de la République colombienne. Réélu deux fois, il a gouverné de 2002 à 2010.

12. Voir http://www.mincit.gov.co/documentos.php?id=168.

13. Ministerio de Ambiente, Vivienda y Desarrollo Territorial, Parques Nacionales Naturales de Colombia, octobre 2006 : http://www.parquesnacionales.gov.co/PNN/portel/ libreria/pdf/CartillaEcoturismoComunitario.pdf. 
institutions décentralisées de l'État, le secteur privé et les communautés dans la mise en œuvre de l'écotourisme. Selon ce document, plusieurs communautés, dont certains groupes au Cabo de la Vela, ont participé à la formulation des axes.

Les personnes interrogées pendant notre visite au Cabo de la Vela, en mai 2006, ont affirmé que le gouvernement de Álvaro Uribe avait accordé des crédits aux populations natives pour aménager des logements. C'est ainsi que s'expliquent la prolifération des kiosques et l'aménagement des rancherías pour recevoir les touristes.

\section{Aspects généraux de l'offre touristique}

Les touristes arrivent pendant l'époque des vacances (décembre, janvier, avril, juin). Ils viennent, principalement, de l'intérieur de la Colombie (des villes comme Bogotá, Medellín, Bucaramanga), de la côte atlantique et, quelquefois, on peut trouver aussi des étrangers (Meisel : 52). Ceux avec lesquels nous avons discuté lors de notre visite, en janvier 2009, visitent le Cabo pour être en contact avec cet environnement "si hostile et si beau ", à l'opposé des grandes villes : ils cherchent le dépaysement, l'éloignement de leur vie quotidienne. D'une manière générale, l'écotourisme à La Guajira met en valeur les paysages, la nature à l'état sauvage et souligne la beauté du désert et des plages ${ }^{14}$. Les touristes sont invités à découvrir la nature et la culture wayuu ${ }^{15}$. La publicité offre de vivre, pour quelques heures ou quelques jours, en milieu naturel et de partager la vie des habitants au cœur des écosystèmes; elle prévient que, à La Guajira, on ne trouvera ni hôtels haut de gamme ni clubs. Pendant notre travail de terrain, en mai 2006 et janvier 2009, nous avons pu constater que des étudiants, des cadres, des intellectuels, des fonctionnaires, bref des touristes d'un statut social moyen, en constituent la clientèle. Ils recherchent un tourisme dit ethnique et écologique où l'autre, le Primo-natif, représente un mode de vie opposé à la modernité : ils ont l'œil curieux pour cet autre différent. Du coup, l'écotourisme et l'ethno-tourisme dans La Guajira établissent des relations de face-à-face entre deux altérités extrêmes : les touristes modernes et cultivés, et les Primo-natifs qui représentent le monde non-moderne. Pour Cazes et Courade (260) «ce face-à-face est toujours une confrontation totale ». Cependant, cette opposition est en mouvement constant car « visiteurs et visités ne sont pas plus réductibles l'un que l'autre à des figures monolithiques aux frontières nettement délimitées. La distinction entre host et guest fléchit bien souvent face aux multiples acteurs

14. Cf. brochure de l'agence touristique Magic Tour Colombia. Voir, aussi, le site : http:// magictourcolombia.com/tours-3/guajira/cabo-de-la-vela/. Consulté en avril 2015.

15. Cf. brochure de l'agence touristique Antioquia de aventura. Accès: http://www. antioquiadeaventura.com/es/planes/318-guajira-cabo-de-la-vela-punta-gallinas-pnnla-macuira.html. Consulté en avril 2015. 
- amateurs, activistes, entrepreneurs culturels, ethnologues... - qui revêtent d'un côté comme de l'autre le rôle de passeurs, d'intermédiaires, occupant une place déterminante dans le développement des activités touristiques » (Doquet et Le Menestrel : 10).

Plusieurs agences touristiques organisent des après-midis et des soirées wayuu et en promeuvent l'attractivité en folklorisant la culture. Elles créent des publicités en mettant en avant la nature, sorte "d'images à vendre " (Cazes et Courade : 250). Voici quelques formules : "Guajira exotique »; "La Guajira est un désert entouré par la mer, le sable, riche en charbon et sel, et peuplé de cactus »; "Guajira magique »" ${ }^{16}$. D'autres agences vont beaucoup plus loin et mettent en avant certains traits culturels des Wayuu et invitent les touristes à observer la danza yonna et manger les plats typiques des Wayuu ${ }^{17}$.

\section{Jepira et le Cabo de la Vela}

Pour certains Wayuu interrogés, le nord du Cabo de la Vela fait partie du long voyage que font les âmes des morts: "quand les Wayuu meurent, leurs âmes font un voyage, elles font un parcours marin, elles vont à Jepira et à la Voie Lactée ». D'autres affirment que "Le Cabo nous amène à Jepira», ou encore «Le Cabo est une sorte de porte qui ouvre sur Jepira ». L'anthropologue Michel Perrin a recueilli des mythes qui relatent que "les âmes des morts " se rendent à Jepira, un lieu imaginaire (1980:31). Chez les Wayuu, le monde des morts est séparé du monde des vivants et selon plusieurs récits, un lieu spécifique au nord du Cabo de la Vela évoque cette séparation. Cet endroit, une portion de terre qui pénètre la mer, désigne une séparation nette des eaux et de la terre. Mais cet espace, pour les Wayuu que nous avons interrogés, « n'est pas tangible ", « c'est une sorte de brouillard sur la mer» et " on ne sait pas où est exactement Jepira ». La relation du Cabo avec Jepira se trouve aussi dans le fait que les éléments « eau » et «terre » sont présents à Jepira. Selon José Enrique Finol Jepira est un lieu où l'eau et la terre convergent (Finol : 33). Certaines agences, à Riohacha, simplifient cette dimension spirituelle en disant que le Cabo de la Vela est un « lieu où demeurent les âmes de personnes mortes $»^{18}$. Ce type de formule

16. Cf. brochures des agences touristiques. Accès : http://www.guajiratours.com/\#!planes-tursticos/c1mwl et http://www.viajaporcolombia.com/sitios-turisticos/la-guajira/. Sites consultés en février 2014.

17. Accès : https://viacoltour.wordpress.com/2010/03/05/guajira/. Site consulté en février 2014.

18. Lors de notre travail de terrain, en 2006, nous avons visité des agences touristiques à Riohacha, comme Luna Guajira, Guajira Tours et quelques opérateurs de transports. Nous avons observé l'abordage des touristes par les fonctionnaires de ces agences et entendu ces commentaires. 
constituait un ingrédient de plus pour attirer les touristes. Les significations de l'au-delà, pour les Wayuu, sont réduites et imprécises. Selon les mythes recueillis par Perrin (1980:29-31), quand l'âme, $a a^{\prime}$ in, est retenue par le monde des esprits, la personne est malade et quand a $a a^{\prime} i n$ part définitivement du corps, la personne est morte. C'est alors que l'âme fait un voyage, avec les objets les plus chers de la personne et rejoint Jepira en passant par la Voie Lactée : là-bas, à Jepira, se trouvent les maisons des morts. Les Wayuu meurent deux fois : la première physiquement, la deuxième à Jepira. Le sens de la mort chez les Wayuu n'est pas évoqué par les agences touristiques. Lors de notre dernier travail de terrain, en février 2015, Cora, une femme wayuu dont nous parlerons plus tard, a affirmé que la question des morts, de l'au-delà, de la signification sacrée de Jepira, ne sont pas des sujets à traiter avec les touristes dans la mesure où ceuxci n'attendent que des informations superficielles.

La culture des Primo-natifs se vend comme une spécialité à explorer et à connaître. Nous avons pu observer, dans certaines rancherías visitées en 2009, et dans d'autres où nous avons séjourné, en février 2015, comme celle d'Ou'liwo (à 15 minutes de Riohacha), le soin apporté par des familles au nettoyage des lieux, à la préparation du terrain pour danser la yonna, au choix des objets artisanaux à présenter aux touristes. La mise en scène de la culture locale s'offre avec prodigalité : théâtralisation culturelle et matérielle, "mise en spectacle " selon les termes de Marc Augé (cité par Cunin et Rinaudo : 154). Dans ce cadre, le tourisme se fonde sur "l'enchantement ", concept qu'Yves Winkin (215) emploie pour marquer la puissance des effets euphoriques du nouvel environnement dans lequel pénètre le touriste.

\section{Les Wayuu, marchands informels du tourisme}

La plupart des propriétaires des agences touristiques de la ville de Riohacha ${ }^{19}$ et d'ailleurs (Medellín, Bogotá) sont des criollos $^{20}$. Ils sont organisés en réseaux

19. Du fait d'être la capitale du département de La Guajira, Riohacha, avec son infrastructure hôtelière et de transport, compte de nombreuses agences de voyage qui permettent aux touristes de mieux préparer leur voyage à la Haute Guajira. Cette ville est considérée, aussi, comme un lieu touristique, grâce à ses plages.

20. À La Guajira, le mot criollo et le mot métis ont la même signification : des individus issus de l'union entre Indiens, Blancs et Noirs. Cependant, des études comme celles de Anne-Marie Losonczy (2008) dévoilent les différences entre la catégorisation de métis et celle de criollo. Affirmant que le terme criollo exprime une autochtonie, une appartenance culturelle, Losonczy montre qu'il a évolué au fil du temps pour exprimer, aujourd'hui, le fait d'être colombien. Ainsi, criollo est surtout le support d'une affirmation culturelle régionale tandis que le terme métis fait référence à des contextes transnationaux, caribéen ou latino-américain (Losonczy, 2008 : 274). 
et emploient des Wayuu qui servent à la fois de conducteurs de voitures pour transporter les touristes et de guides culturels. L'offre des guides wayuu constitue un plus lié à la prétention des agences de donner accès à de l'authentique. D'ailleurs, des familles wayuu font partie du réseau des agences et offrent leur demeure pour y montrer des danses et de la cuisine wayuu, et quelquefois des rituels chamaniques. D'après Franklin, criollo guajiro, propriétaire d'une agence de tourisme à Riohacha,

Los Wayúu son las personas más aptas para transportar los turistas porque ellos conocen las trochas, sobretodo en invierno. Ellos saben por dónde pasar, conocen muy bien la trilla, la fresquita porque tú sabes que hay muchas trillas, unas sobre otras y eso confunde. Ellos conocen todo el desierto y ¿quién mejor que ellos para explicar su cultura? Muchas agencias hacen eso. (entretien, Riohacha, juin 2006. Karen López).

Cependant, des ethno-métis ${ }^{21}$ et des Wayuu parviennent à acquérir un $4 \times 4$ pour offrir directement aux touristes leurs services de transport et se passer des agences.

Mais si le Cabo est un des endroits les plus vantés, le village de Nazareth devient plus exotique encore car, pour arriver jusque-là, les touristes doivent effectuer un voyage à travers la topographie accidentée du désert. À Nazareth, plusieurs rancherías ou maisons de Wayuu sont adaptées pour loger les touristes avec des lits ou des chinchorros (hamacs), et certaines comptent des groupes électrogènes; il y a des toilettes et une salle de bains artisanale avec des seaux remplis d'eau. Elles offrent aussi de la nourriture. Les touristes arrivent avec l'objectif de visiter la Serranía de la Makuira, Parc National Makuira depuis $1977^{22}$. Son bureau à Nazareth a établi un parcours touristique payant et les guides sont de jeunes hommes et des jeunes femmes wayuu qui ont reçu une formation au Parc National pour expliquer la flore et la faune, et présenter les endroits mythiques de la culture wayuu dans la Serranía.

21. Terme employé par nous pour distinguer les Wayuu qui sont nés d'une mère wayuu et d'un père non-wayuu mais qui se sont éloignés de la culture wayuu : la plupart des ethno-métis habitent dans les villes et sont intégrés à la vie sociale, économique et politique de la société locale comme un criollo. Néanmoins, il convient de préciser que, pour les Wayuu, le fait d'être né d'une mère wayuu suffit pour être Wayuu même si la personne concernée ne parle pas la langue ni ne pratique les coutumes wayuu, ni n'habite sur le territoire. Leoncio, Boni, et Carlos, Wayuu dont nous parlerons ensuite, sont nées d'une mère wayuu et d'un père non-wayuu.

22. Voir le site Parques Nacionales de Colombia. Accès : http://www.parquesnacionales. gov.co/portal/eMakuira s/ecoturismo/region-caribe/parque-nacional-natural-macui$\mathrm{ra} /$. 
Pour situer le cadre socioéconomique dans lequel se déroule une partie de notre histoire, nous allons décrire l'offre variée de logements touristiques que les Wayuu proposent. Ceci nous permettra d'évaluer les réactions des touristes dans notre histoire et le type de représentations qu'ils se font de l'accueil wayuu. De même, nous pourrons voir quelles représentations se font les Wayuu de leurs clients.

L'offre de logements touristiques wayuu est variée. Pour comprendre cette variété, nous allons prendre trois exemples : Cora, Boni et Carlos. Cora, une Wayuu de 50 ans, qui habite depuis toujours au Cabo, a un bungalow où les gens peuvent loger et manger. Le logement de Cora est simple : un rancho de deux chambres construit en torchis. Les touristes dorment, en moyenne, quatre par chambre, dans des hamacs. La douche se prend avec un seau qu'il faut remplir à l'extérieur. Cora obtient de l'eau d'un carrotanque, un camion qui la distribue. La nuit, chez Cora, coûte 2 euros. Elle affirme : "Aquí estamos bien, tenemos trabajo. Aquí estamos organizados en el Cabo. Hay gente, empresas que quieren construir hoteles cinco estrellas pero nosotros no los dejamos, no nos dejamos ». (entretien, Cabo de la Vela, mai 2006. Karen López). Elle explique encore que « varios Wayuu vivían antes en otros lados y ellos se regresaron para aprovechar el programa de Uribe y hacer su enramada porque eso es trabajo. Mira el caso de mi sobrino, él vivía en Maracaibo y no tenía trabajo allá y hoy él tiene su negocio aquí, por allá más lejos, él vende pescado, arroz, cerveza». (idem)

Cora est une femme reconnue et respectée au Cabo et dans d'autres villes de La Guajira. Les touristes qui arrivent chez elle sont recommandés par ses amies. Pour elle, le logement des Wayuu doit rester tel qu'il est : "Podemos hacer algunos arreglos a la ranchería para que los turistas se sientan bien pero no hay que cambiar todo, porque es nuestra cultura, ni hacer hoteles; aqui no queremos hoteles de 5 estrellas. » (idem) Pour Cora, la valeur la plus importante à offrir aux touristes est la culture même et "s'ils viennent ici [au Cabo] ils doivent nous accepter tels que nous sommes» (traduction personnelle). Elle attend que les touristes viennent, connaissent et valorisent la culture wayuu. En janvier 2009, lorsque nous avons rendu visite à Cora, nous avons pu constater qu'elle louait une de ses chambres.

Boni a deux bungalows avec deux petites chambres construites en yotojoro, le cour du cactus, et on peut dormir dans un lit; il y a aussi un lavabo, un seau rempli d'eau, une lampe et une terrasse avec trois chaises. Dans chaque chambre peuvent loger deux touristes. Boni n'a pas de groupe électrogène et il vante l'idée que la nature est plus belle à vivre sans électricité ni eau potable. Une nuitée, chez Boni, coûte 15 euros. Le jour où nous lui avons rendu visite, en janvier 2009, il n'y avait pas de touristes. Voici un extrait de son récit recueilli, en mai 2006 : "Me dices que aquí es caro. Voy a explicarte que esto no es caro, ¿tú ves allá donde está la luz? Allá hay hoteles más barato pero ¿quién va dormir allá 
si hay tanto ruido porque pone música toda la noche? Nadie. Aquí estamos con la naturaleza, aquí es tranquilo y aquí la gente paga con gusto. " (entretien, Cabo de la Vela, mai 2006. Karen López)

Boni croit que les touristes recherchent des endroits où vivre la nature à l'état sauvage. Mais son discours évoque aussi l'image ancienne du Cabo où l'isolement faisait de cet endroit un lieu "magique " pour les rares touristes qui le visitaient. Boni ne mobilise pas les atouts culturels mais ceux de la nature. Pour cet homme de 60 ans qui a grandi et habité dans la ville de Riohacha, avoir ce logement au Cabo est une chance, car il était au chômage et il n'a pas de retraite : "Cuando supe que el gobierno daba préstamos para construir quioscos yo ví que había que aprovechar para salir de este atolladero. » (idem)

Le bungalow de yotojoro de Carlos, un Wayuu de 35 ans, est, pour sa part, situé dans la partie éclairée du Cabo. Face à la plage, Carlos offre des logements et de la nourriture et il a des kiosques pour vendre des jus. Il a des lits et des chinchorros et il peut accueillir dix touristes. Son gîte est éclairé grâce à un groupe électrogène. La nuit, chez Carlos, coûte à peu près entre 7 et 10 euros, selon que le touriste préfère le lit ou le chinchorro. Carlos est marié avec une criolla du département de Córdoba, et il habitait, avant, la ville de Maicao. Il s'est installé quand le Président Uribe a promu la construction de logements touristiques au Cabo. La femme de Carlos a orienté celui-ci vers le concept touristique de logement et de kiosques. Le logement de Carlos était presque complet quand nous l'avons visité, lors de ce voyage en janvier 2009.

Dans les trois exemples présentés, nous pouvons percevoir comment chacun vit la culture wayuu. Boni et Carlos sont des Wayuu urbanisés qui ont vécu loin de leur territoire ancestral ; ils sont retournés au Cabo pour échapper au chômage, ne parlent pas wayuunaiki, se sont mariés avec des non-Wayuu et ne pratiquent pas les traditions wayuu. Cora, par contre, est une Wayuu qui a grandi toute sa vie dans le Cabo, parle wayuunaiki, pratique les coutumes wayuu et défend sa culture. Pour Boni et Carlos, le fait de ne pas vivre à fond la culture n'est pas un handicap, ni un défaut. Ils se sentent wayuu à part entière car, pour eux, le fait d'être fils de femme wayuu est suffisant pour faire valoir leurs droits de vivre sur le territoire. Ils pensent que connaitre la culture non-wayuu est un avantage pour leur négoce touristique ; et ils définissent leur négoce d'après ce qu'ils pensent sur les touristes.

Carlos, Boni et Cora possèdent leur représentation des touristes et de ce qui est en mesure, selon eux, de les attirer. Le fait que le logement de Carlos soit le plus sollicité23 indiquerait que les touristes veulent séjourner dans un minimum de confort. L'idée de trouver une nature ou une culture très riches dépourvue

23. Cette observation a été confirmée à l'occasion de plusieurs visites, entre 2010 et 2012. 
de tout confort peut devenir un obstacle au voyage. C'est aussi un inconvénient pour les Wayuu dont les logements ne sont pas considérés comme confortables. Les différences de représentation existant entre les trois Wayuu montrent les degrés d'accessibilité des Wayuu à la chaîne économique touristique. Les Wayuu fixent leur prix à leur gré. Ainsi, le manque d'électricité chez Boni constitue, pour lui, un atout pour augmenter ses tarifs tandis que pour Carlos, c'est un élément inconfortable majeur qui peut menacer son offre touristique ; autant l'un que l'autre le contournent selon la représentation qu'ils se font du marché touristique. Autrement dit, le fait d'avoir ou de ne pas avoir d'électricité est un avantage sur lequel chacun argumente en sa faveur. Nous pouvons dire que pour Boni, celui dont le prix est le plus élevé, les touristes ont la capacité de payer la somme demandée et il ne fait ni réductions ni ne négocie, selon ses propres aveux. Carlos est celui qui a plus d'expérience dans le domaine touristique du fait d'avoir vécu dans une autre région touristique. Il choisit le créneau de prix moyen pour maintenir une clientèle. Cora, pour sa part, marque de manière radicale, par son prix très bas, qu'elle n'est pas intéressée par l'argent. Elle met l'accent sur la culture wayuu et le prix de son logement contraste avec celui de Boni et de Carlos.

\section{Récit de voyage touristique : diverses images à collecter et à décrypter}

Parmi les touristes qui arrivent à La Guajira, certains préfèrent faire le voyage directement avec des guides locaux, sans l'intermédiaire des agences. Nous allons exposer, dans les lignes qui suivent, notre propre expérience touristique.

À la demande d'une personne originaire de Bogotá mais habitant l'île de La Réunion, et dont nous venions de faire la connaissance, nous avons organisé un voyage en Haute Guajira colombienne, en janvier 2009. Le protagoniste de notre histoire a manifesté son désir ancien de connaître La Guajira, en compagnie de son frère. Il nous a expliqué qu'il avait le projet de voyager dans cette région en janvier 2009. Curieusement, nous allions aussi visiter La Guajira à ce moment-là. Nous retrouver dans ce projet pour la même période ouvrait la possibilité de faire le voyage ensemble. Pour eux, c'était un avantage de connaître La Guajira avec quelqu'un de la région. De notre côté, ce n'était pas la première fois que nous pouvions faire le guide. Notre service n'était pas payant.

Cette personne a magnifié La Guajira devant nous, « cette belle terre mystérieuse avec son désert et la culture des indigènes ». Les deux frères étaient photographes, l'un professionnel, l'autre amateur. Nous cherchions un guide wayuu pour faire un circuit de 5 jours. Une relation (personnelle) nous a conseillé Leoncio, un ethno-métis qui habite Riohacha et a des parents wayuu dans la 
Haute Guajira, possède un $4 \times 4$ et fait le transport de touristes. Leoncio a accepté de nous amener en Haute Guajira. Nous sommes partis vers Taroa, Punta Gallinas (le point le plus septentrional de l'Amérique du Sud), et La Unión. Leoncio a des parents sur ce territoire. À Punta Gallinas, il nous a amenés chez Marianella, une cousine à lui. À notre arrivée, un des photographes nous a prévenus : "Il faut faire attention avec vos appareils de photos car il y a des voleurs ", en faisant référence aux Wayuu. Nous y sommes restés deux nuits. Le soir, les frères de Bogotá ont offert à Leoncio de la bière et ont fait preuve de gentillesse envers lui. Tout au long du voyage, les touristes ont pris des photos des Wayuu : femmes, enfants, hommes, la plupart membres de la famille de Leoncio. Un des photographes a avoué qu'il prenait des photos pour les vendre aux collectionneurs. Ils mettent en scène les Wayuu devant un décor, souvent très simple comme un tissu de couleur brune destiné à effacer le paysage, puis ils leur demandent de s'habiller avec leurs habits typiques. Les photographes cherchaient des caractéristiques physiques des Wayuu (des personnes âgées, des visages particuliers, des jeunes femmes) pour en faire un produit avec tous les atouts de l'exotisme et les revendre à des revues spécialisées.

Le lendemain, nous nous sommes dirigés vers Nazareth où nous sommes arrivés vers 22 heures. Nous (qui écrivons ce récit), avons proposé de loger dans la famille Medina, que nous connaissons depuis longtemps. Nous avons suggéré aux deux frères de ne pas demander de repas vu que la famille avait déjà fermé la cuisine. Notre décision de loger chez les Medina a mis mal à l'aise Leoncio. Bien que nous ayons pris conscience de son désaccord silencieux, nous n'en avons pas compris tout de suite les raisons. Leoncio a décidé de chercher un autre endroit où dormir. Le lendemain, après un parcours au Parc Makuira, les hommes de Bogotá ont bu de la bière avec Leoncio. Ils sont arrivés chez les Medina vers minuit avec une caisse de bière pour l'offrir aux hommes de la maison. Le chef de la famille a refusé de boire. Le jour suivant, la tension dans le groupe a émergé sans raison apparente à nos yeux. Les hommes de Bogotá ont manifesté une contrariété et une déception vis-à-vis de Nazareth et du Parc Makuira. Ils ont imposé d'aller immédiatement au Cabo de la Vela, leur destination de prédilection. À la sortie d'un village, la voiture s'est embourbée du fait de la perte, par Leoncio, de son habileté de chauffeur, après l'ingestion de grandes quantités de bière avec les touristes. Pour sortir de cette impasse, nous avons cherché de l'aide dans une ranchería qui se trouvait dans les alentours. La famille que nous avons rencontrée nous a aidés sans demander aucune prestation en retour.

À notre arrivée au Cabo, Leoncio faisait corps avec les photographes qui prirent leur distance vis-à-vis de notre personne. La situation s'est dégradée et le soir même la dispute a éclaté dans le groupe et les deux frères, avec le 
guide, ont décidé de loger chez Carlos et nous-même, de loger chez Cora. Les touristes ont manifesté leur mécontentement en signalant que la famille Medina avait demandé un prix très élevé par rapport à celui qu'ils attendaient et qu'en plus, quand ils sont arrivés, nous avions empêché qu'ils fassent la cuisine pour eux. Ils ont ajouté que la famille Medina ne les avait pas bien accueillis et qu'ils avaient mal dormi. D'ailleurs, ils ont manifesté qu'ils étaient déçus de toute La Guajira, du Cabo même car ils ne pouvaient pas faire de la plongée. Le voyage arrivait à sa fin avant la date prévue car les conflits ont continué. Deux jours plus tard, nous avons rencontré, par hasard, trois hommes de la famille Medina, à Riohacha, et nous leur avons raconté l'histoire. Ils nous ont expliqué que Leoncio ne loge jamais chez eux, car il réclame toujours une commission au maître de la maison pour amener des touristes, ce que les Medina n'acceptent pas. À Nazareth, il n'y a qu'une personne, un parent de Leoncio, qui accepte ces conditions. D'après Tico Medina, Leoncio est quelqu'un qui veut tirer des bénéfices de tout.

Cette expérience est révélatrice, à nos yeux, pour comprendre l'imaginaire dialogique d'un groupe d'acteurs impliqués dans un projet touristique: des touristes, comme ces deux frères qui cherchaient à harmoniser leur voyage touristique avec un objectif professionnel concret ; un guide natif qui cherchait à consolider sa clientèle et nous qui faisions le pont entre les uns et les autres.

Tout d'abord, ces hommes de Bogotá sont un exemple d'hommes et de femmes faisant le voyage à La Guajira sous couvert de pratiquer un tourisme écologique ou ethnique ; en fait, ils viennent pour accomplir un travail spécifique. Au cours de nos années passées là-bas, nous avons été témoin de cette particularité des touristes ${ }^{24}$. À travers des amis qui recommandaient à leur tour des amis, nous avions été plusieurs fois sollicitées pour le service de guide. Notre cas est extensible à d'autres parents et d'autres amis et nous pouvons croire que ce type de service, fait sous l'égide de l'amitié, ou d'une possible amitié, est devenu une habitude. Or, ce type de touristes voit La Guajira et les Wayuu comme un objet de profit et pas simplement comme un objet de curiosité : ils arrivent avec un but déterminé et la construction de relations avec les Natifs et les Primo-natifs est préfabriquée, faite de représentations qu'ils n'ont pas été portés à interroger spontanément. Construire la relation n'est pas leur objectif premier. L'occultation de l'objectif à atteindre par les deux frères (constituer une collection de photos à vendre), dans le cas exposé, annonce déjà un terrain susceptible de "malentendus».

Pour les hommes de notre histoire, l'image de la tâche de ce voyage était d'aboutir à trouver des personnes répondant à des critères stéréotypés à

24. À notre connaissance, il n'y a pas d'études sur ce sujet. 
photographier, d'après l'analyse a posteriori que nous sommes en mesure de tirer de cette expérience. La Guajira et ses habitants représentent encore une région éloignée des centres du pouvoir, sauvage, exotique et digne d'être photographiée, peinte, analysée et présentée au reste du monde. Pendant le voyage, un des photographes m'a fait part de son désaccord sur la manière de vivre des Wayuu : sans énergie électrique, sans eau potable, sans route, sans hôtel cinq étoiles : "le confort et la culture indigène ne sont pas incompatibles. La responsabilité d'autant de retard dans le développement revient aux politiques". Ce qu'attendait notre protagoniste était un confort garanti à tout moment pour déguster sans gêne la culture de l'autre. Bien qu'il fût prévenu sur l'inconfort de l'endroit, il regrettait ces manques.

Les paroles prononcées par un des photographes accusant de voleurs la population native dévoilent l'image qu'il se fait de cet autre différent, un voleur potentiel dont il faut se méfier, et met en scène la peur banale du touriste. Sa place dialogique de touriste s'engouffre dans une présentation de soi comme proie des Wayuu, victime pressentie du fait de sa nature d'étranger. Ce faisant, il se donne le droit de photographier la personne native sans la rétribuer, ce qui l'amène à entrer dans le rôle de prédateur d'images à collectionner. Conscient qu'il ne peut opérer qu'avec l'appui d'un Natif influent, il assigne à celui-ci le rôle d'arrangeur, apte à convaincre les membres de son lignage de la légitimité de la prédation. Pourtant, ce n'est pas le voleur qui est photographié, mais le Natif.

La représentation de La Guajira, y compris de sa population, telle qu'elle est construite par ces hommes, est celle d'une région arriérée, éloignée du monde, du développement, du progrès. Cette vision suppose que sa valeur ne peut que venir des autres, des étrangers, sous la forme d'une aide condescendante aux Primo-natifs à qui l'on apporte des subsides en payant sa nourriture et son logement. En montrant les traits saillants de ce monde exotique, voire une "scène ethnique " (Alvarado Pérez $2001: 21$ ), le photographe joue un rôle de mécène, toujours sous couvert d'aide envers son prochain; il met en spectacle une population marginalisée, selon les normes requises de ce qui est une image photographique. Autrement dit, la photographie, comme "système conventionnel ", comporte des normes requises sur l'esthétique de l'image photographique (Alvarado Pérez 2009 : 15). « Nous allons montrer la beauté des Wayuu ", "Les femmes wayuu ont cette expression de sérénité, de noblesse, c'est ça que nous photographions " affirmaient-ils. Ces images sont recherchées par une clientèle de collectionneurs, de mécènes dont la représentation des Peuples natifs est stéréotypée. Le modèle de la mise en scène des photographes est proche des photographies faites au XIX ${ }^{\mathrm{e}}$ siècle et au début $\mathrm{du} \mathrm{xx}^{\mathrm{e}}$ siècle, où des visages, des expressions, des gestes ont été 
répertoriés pour exhiber cet autre différent lors des expositions humaines en Europe $^{25}$.

Dans cette logique, et en tenant compte du fait que nous sommes originaire de La Guajira, la relation qui pouvait s'établir entre eux et nous était nécessairement une relation inégale, où eux jouaient le rôle d'hommes modernes, développés, cultivés et nous-même, en tant que Métisse guajira, l'opposé. Ainsi, ces hommes ont souligné, en première instance, leur ascendance espagnole, en disant toute leur fierté d'avoir des arrière-grands-parents venus d'Espagne. Pourtant, ces deux frères, tous deux Colombiens comme nous, ont, comme nous, une part de Primo-natifs dans leur généalogie et, à ce titre, appartiennent à ce monde. Les deux frères touristes sont, en effet, des Métis par leur grand-mère primo-native : en effet, au cours de la matinée passée dans le Parc Makuira, l'un d'eux a manifesté l'existence de cette part de lui-même avec laquelle il reprenait contact dans cette nature si belle. Par cette révélation, il marquait fortement son appartenance à ce monde, motivation possible du voyage. Là, au milieu de la beauté de la nature, loin de ces cercles sociaux criollos, cette reconnaissance de l'origine ancestrale ne pouvait pas être combattue. Cette situation révèle que les deux frères laissaient leur place dialogique de touristes pour prendre, pour un instant, celle des Natifs, de descendants de Primo-natifs. À ce moment-là, ils se fondaient avec cet autre différent. D'ailleurs, la situation où les photographes partagent l'ingestion d'alcool, jusqu'à l'ébriété, avec Leoncio, peut signifier cette communion entre les deux touristes et le guide. Par ailleurs, le fait qu'ils aient offert tant de bière à Leoncio met en évidence que les touristes de notre histoire voulaient nous écarter de la relation qu'ils pouvaient créer avec lui : en lui offrant cet alcool, ils établissaient un accord tacite de complicité avec lui. Les frères cherchaient à garantir l'accès aux personnes à photographier. En effet, le fait que Leoncio soit Wayuu le légitimait à leurs yeux comme source d'informations sur la culture wayuu, tout en nous disqualifiant, en tant que Métisse comme eux. Même l'offre de la caisse de bière à la famille Medina exprime le désir des deux frères d'étendre la complicité trouvée avec Leoncio. Pour un des frères, l'attribut de gentillesse propre à cette place dialogique s'imposait de lui-même, mais indiquait en même temps sa méconnaissance de la manière d'être gentil dans la culture wayuu.

La beauté de la nature offerte par le Parc Makuira est oubliée rapidement quand un des frères décide de faire de la plongée au Cabo. En réalité, dans la Haute Guajira, les vents sont forts et la mer est agitée. Une des caractéristiques géographiques du Cabo est la force des courants qui rendent difficile la navigation. Il n'y a pas de lagons. D’ailleurs, «le marketing territorial » des

25. Voir, par exemple, le catalogue de l'exposition Exhibitions. L'Invention du sauvage, Musée du Quai Branly, 2011 (bibliographie en fin d'article). 
agences touristiques ne mentionne pas la possibilité de faire de la plongée. Toutefois, pour le touriste en question, faire de la plongée était une perspective qui allait de soi car il était habitué à cette activité sur l'île de La Réunion. Dans sa représentation, l'absence des sports de plage est liée au sous-développement de La Guajira. Le "malentendu », dans ce cas, n'est pas produit par l'offre touristique mais par l'histoire de la personne et de ses préjugés en ce qui concerne ce qu'est la côte dans un milieu touristique.

L'image de la tâche de Leoncio que nous nous faisions (les deux touristes et nous-même) était, au départ, claire : elle consistait à présupposer son efficacité autant comme chauffeur sur terrain accidenté que comme guide de sa culture. La position spécifique de Leoncio, à savoir se solidariser avec les touristes, pourrait s'expliquer par le fait qu'il voulait les garder comme clients en se passant de notre intermédiaire : il pouvait anticiper sur le retour de ces photographes et sur le bouche-à-oreille pour sa promotion. D'ailleurs, Leoncio construit sa clientèle de cette façon, car il n'a pas de bureau, pas d'agence, pas de publicité ; l'information circule parmi les amis : ce sont eux qui transmettent ses coordonnées. Par ailleurs, Leoncio a compris qu'il ne pouvait pas influencer notre décision sur les endroits où loger, car nous avons notre réseau d'amis wayuu, ce qui représentait, pour lui, un manque à gagner. Leoncio a profité de la dispute pour nous écarter de la relation qu'il voulait construire avec les touristes. Mais ce qui est clair dans la compréhension du comportement de Leoncio c'est que, chez les Wayuu, le réseau familial domine en tant que clans corporatifs. Ainsi, le guide ethno-métis ne cherche pas à amener des profits aux familles autres que celles qui appartiennent à son lignage. Dans ce sens, il est compréhensible que Leoncio ait cherché un autre endroit, celui d'un parent, où loger. S'il avait décidé de loger chez les Medina, peut-être que sa place dialogique, à ce moment-là, aurait été celle d'un touriste?

Il est nécessaire de faire remarquer que, dans ce type de voyage, la présence du guide est fondamentale et que chaque membre du groupe établit une relation avec lui. C'est à travers sa médiation que les touristes, ou visiteurs, accèdent à la connaissance des régions ou des cultures. Le touriste se déplace toujours avec " son double », terme employé par Yves Winkin pour expliquer le rôle du guide : « Il [le guide] est celui qui non seulement médie le regard du touriste, s'interpose entre celui-ci et la réalité mais encore participe activement à la construction de cette réalité, en fournissant à son "client» des cadres de perception et des signes d'accréditation de l'univers qu'il découvre» (Winkin : 222).

Si le guide est le médiateur entre le groupe de touristes et les Wayuu, cette médiation peut être considérée, dans notre histoire, comme partagée car nous étions aussi médiatrices, rôle qui pouvait perturber Leoncio dans la mesure où il nous ressentait en compétition avec lui dans les décisions de logement. De 
cette façon, la place dialogique que nous avions, comme concepteur du voyage, a été menacée par Leoncio, principalement du fait de son efficacité pour réussir à satisfaire les objectifs des touristes.

Mais l'analyse de cette expérience ne peut être complète si nous n'examinons pas notre propre comportement. L'image de notre tâche était celle de proposer une médiation entre les deux touristes et la culture locale. D'ailleurs, pour nous, offrir nos services dans la gratuité trouvait sa récompense dans le plaisir de faire connaître La Guajira et plus particulièrement la culture wayuu, ce qui s'harmonisait avec nos habitudes anciennes. À cela, s'ajoutait le fait que, habitant à ce moment-là à La Réunion, nous envisagions de poursuivre nos échanges avec un des photographes. Peut-être voulions-nous construire une amitié pour partager des connaissances, des préoccupations anthropologiques dans ce coin du monde.

Cependant, interpréter les gestes et les paroles de ces deux photographes comme relevant de représentations négatives et stéréotypées a peut-être été, chez nous, le motif déclencheur d'une forme de révolte. Nous les avons vus comme des étrangers affamés d'exotisme et d'images de Primo-natifs à commercialiser : l'ont-ils senti ? Eux et nous sommes peut-être tombés dans un affrontement inconscient où chacun reprenait à son compte l'histoire tissée entre les habitants des centres de pouvoir, les grandes villes comme Bogotá, et les habitants de la périphérie marginalisée, comme La Guajira. Le fait d'avoir choisi un logement séparé au Cabo de la Vela exprime d'ailleurs la représentation que chacun avait sur l'autre différent. Nous avons choisi Cora qui, à nos yeux, était plus proche de la culture wayuu et plus éloignée du bruit et de la foule. Une interrogation demeure : à quel moment avons-nous occupé la place dialogique de touriste? Avons-nous été poussées à occuper cette place par les deux touristes? Au moment où les deux photographes imposaient leur décision de changer le programme du voyage et de partir de Nazareth au Cabo de la Vela, ils ont pris la place de concepteur de voyage et nous celle de touriste. L'image de cette situation a aggravé le conflit au point que nous avons préféré abandonner le groupe, un peu plus tard.

L'expérience de ce voyage nous permet d'envisager l'idée que le tourisme que nous avons décrit est une suite de situations en mouvement permanent. Les places ou les rôles établis ne sont pas statiques : les touristes, les guides, les concepteurs de voyage et d'autres rôles qui émergent au fur et à mesure des interactions peuvent changer en continu. Alors, comment se joue finalement le lien entre touristes et population native? La transaction de service, ou de commerce, finit par déterminer la relation dans un voyage touristique : l'un est le client et l'autre doit répondre à ses attentes, autant au niveau du confort qu'au niveau culturel. Nous avons vu que les protagonistes photographes de 
notre histoire exprimèrent une plainte à propos des prix, qu'ils jugèrent élevés, du service offert par la famille Medina et d'autres Wayuu. Pour éclaircir cette image que les touristes avaient retenue, nous allons expliquer le mouvement microéconomique de ce séjour touristique.

\section{«L'effet goutte d'eau », l'écart des attentes confrontées au marché}

Tout d'abord, la dynamique économique de l'activité touristique en réseau produit une circulation d'argent dont les grosses sommes restent chez les intermédiaires, avant d'arriver à destination. Pour étayer cette affirmation, nous allons prendre en compte l'histoire des deux photographes, car ce cas illustre ce que nous affirmons.

Souvenons-nous qu'un de ces deux hommes habite à Bogotá et l'autre sur l'île de La Réunion, dans l'Océan Indien. Ce dernier a dépensé des sommes importantes pour prendre l'avion depuis La Réunion jusqu'à Bogotá et arriver finalement à Riohacha; coût de l'aller-retour : 1600 euros environ ${ }^{26}$. Puis, à Riohacha, il a payé l'hôtel, les dépenses de nourriture, les achats en boutique pendant deux jours : à peu près 110 euros ; ensuite, le coût du transport payé à Leoncio pour 4 nuits et 5 jours : presque 180 euros. Les dépenses chez les Wayuu ont atteint la somme d'environ 100 euros, pour l'ensemble du parcours ${ }^{27}$. Les dépenses des deux frères, pour deux nuits et 4 repas, ont été d'environ 49 euros (l'un d'eux a choisi de dormir dans le lit). À cela s'est ajouté le retour de Riohacha à Bogotá. Au total, il a dû débourser environ 2090 euros. Pour l'autre homme, ce coût était peut-être de 680 euros. Du coup, quand le photographe de La Réunion a dû payer le prix demandé par la famille wayuu à Nazareth, il a trouvé cela très cher.

C'est ce que nous appelons «l'effet goutte d'eau ", eu égard à l'expression "c'est la goutte d'eau qui fait déborder le vase ». À force de payer des sommes élevées pendant le voyage, le touriste arrivé à destination explose et croit que les Primo-natifs l'exploitent. Bien que cette situation ne se soit pas produite avec l'autre photographe, celui-ci a adhéré à l'idée de son frère. La discussion autour des prix demandés par les Wayuu a provoqué une sorte de méfiance chez les photographes à l'égard des Primo-natifs : chaque fois qu'ils devaient payer pour leurs repas, ils se méfiaient et ils recomptaient la monnaie en craignant d'être volés. Il y a eu cristallisation de la peur d'être exploité. Nous avons le sentiment que la peur d'être volé, relayée par les accusations de vol, exprime la manière de voyager de ces deux hommes.

26. Prix approximatif en 2009.

27. Les tarifs, chez les Medina, étaient les suivants : 8 euros le lit, 6,50 euros le chinchorro, 5 euros par repas. 
Mais on pourrait considérer que la dépense de grosses sommes se passe ailleurs : avec les lignes aériennes, les hôtels, les restaurants et les boutiques en ville, là où leur révolte ne trouve personne à qui parler ; avec les entreprises, les relations sont mises à distance par la technologie et l'administration. Si nous suivons les étapes des deux frères dans leur parcours touristique, les premiers qui font de gros bénéfices sont les grandes entreprises, comme les lignes aériennes, tant du pays d'accueil que des pays dits développés. Dans l'échelle de dépenses effectuées pour nos protagonistes, la deuxième place (de bénéficiaires de ces dépenses) revient aux entreprises de taille nationale du pays d'accueil; la troisième place revient aux entreprises des localités, comme les hôtels et les restaurants. À la quatrième place on trouve le guide natif pour ses services de transport et, en dernière position, les propriétaires de logements et de restaurants wayuu. Dans cette logique, nous rejoignons Naïri Nahapétian et Georges Cazes quand ils affirment que "le tourisme enrichit surtout les plus riches".

Ainsi, tout au long du parcours, depuis leur lieu de résidence jusqu'à la destination finale, ces touristes tolèrent des dépenses lourdes, en les acceptant avec dignité ; puis, arrivés près de l'objectif qu'ils se sont fixé, ils sont débordés par une demande de dépense supplémentaire, et commencent à négocier des réductions de prix et à se montrer soupçonneux : c'est « l'effet goutte d'eau ». C'est la conséquence du face-à-face visé, désiré avec ceux qu'ils veulent rencontrer. Dans ce lieu longtemps imaginé dans son exotisme, l'argent ne circule pas dans les mêmes volumes et ne répond plus aux habitudes d'évaluation du sujet.

Sachant que chez les Wayuu, il ne s'agit pas d'un tourisme de haut niveau, sans doute les touristes s'attendaient-ils à des prix exagérément bas associés, confusément, à l'image de gratuité symbolisant la fin du marché ou l'impossibilité pour ces populations d'entrer dans le marché. Mais c'est l'irruption des besoins réels qui sanctionne l'image de gratuité : les touristes ont besoin de manger, de dormir, de se déplacer en voiture et les Wayuu sont là pour les satisfaire en contrepartie de revenus, pour combler leurs propres besoins. Cependant, nous pouvons supposer que, pour les touristes, le besoin réel des Wayuu de recevoir des revenus du marché touristique est effacé : l'image que les Primonatifs peuvent vivre hors du marché semble ancrée dans la représentation de ces touristes pourtant avisés. Tout se passe comme s'ils voulaient une gratuité comme celle obtenue dans leur rôle dialogique de photographe, où la prise de photos a été accordée libre de droits par les Wayuu.

Mais, les Wayuu sont bien incorporés dans la dynamique économique. Cela veut-il dire que les Wayuu sont doués pour le marché ? C'est une raison invoquée par nos protagonistes pour se méfier d'eux. Ce paradoxe n'existe que parce que la représentation du Natif voleur est restée figée chez les photographes. Il est vraisemblable, d'après notre histoire, que, si les représentations voyagent 
avec les voyageurs, l'imaginaire dialogique nous montre que celles-là changent relativement peu.

On pourrait considérer que la population ciblée, au même titre que son territoire, est en permanence "travaillée " au nom du développement, mais sa préparation très relative à l'accueil des étrangers justifie magistralement qu'elle ne reçoive, du fait de son existence hors-norme, qu'une faible part de la rente touristique. Le paradoxe sert l'ensemble des prestations périphériques qui vont se trouver mieux rémunérées. Il est vraisemblable que la cible photographique des touristes, ici les Wayuu, considérée comme mal préparée, jamais parfaite, mais pourtant tellement "serviable ", pourra demeurer dans une situation intermédiaire, à la limite de l'acceptabilité du client qui croira, dans l'effort qu'il fait pour s'adapter, recourir à la toute-puissance de sa subjectivité. Le fait de faire un effort pour s'adapter ne justifie-t-il pas la faible rémunération du prestataire d'un service en dessous du confort standard?

D'après ce que nous avons pu observer pendant notre séjour au Cabo et à Nazareth, les Wayuu que nous avons rencontrés, et qui travaillent dans le tourisme, perçoivent des revenus qui peuvent devenir importants s'ils sont complétés par d'autres sources que le tourisme. Considérant celui-ci comme une activité saisonnière, ils combinent cette activité avec le commerce, l'artisanat, le transport. La particularité de cette activité chez les Wayuu (offrir des services chez eux) permet que sa réalisation soit assumée par la famille entière, où les femmes jouent un rôle très important, en économisant sur d'autres dépenses comme celle d'engager du personnel : les femmes font la cuisine et le ménage pour les touristes, et les autres membres de la famille aident à remplir les réservoirs d'eau, à préparer les chinchorros, etc. Avec l'argent de cette activité, la famille achète des animaux - des chèvres -, fait des aménagements dans sa maison avec de nouveaux kiosques ou enramadas, achète des provisions, notamment afin de garantir les services aux touristes.

Dans la famille Medina, la fille travaille comme institutrice dans une école de Primo-natifs et deux de ses frères travaillent aussi dans des institutions publiques. Le père cultive de petites surfaces. Dans le hameau qu'elle habite, cette famille est respectée mais n'est pas considérée comme riche, dans le sens wayuu (voir Introduction, ci-dessus). Marinelle, la cousine de Leoncio, à Punta Gallinas, gère son budget de la même façon : elle est institutrice et elle s'occupe des touristes en faisant la cuisine et le ménage ; sa famille n'est pas riche. Cora, dans le Cabo de la Vela, a des employés : deux jeunes filles qui font la cuisine et un jeune homme qui assume les fonctions de serveur au restaurant. Tous sont Wayuu, ils travaillent en échange de nourriture et d'un logement. Avec ses revenus, Cora fait des investissements dans sa ranchería et achète des animaux. Ainsi, ces Wayuu qui se consacrent au tourisme en tirent des profits variables, 
les uns plus que les autres en fonction du village et des réseaux de chauffeurs qui peuvent leur amener des touristes. D'après nos observations, il est vraisemblable que les hiérarchies sociales, chez les Wayuu, restent indépendantes de l'activité touristique, mais celles-là peuvent être modifiées en vertu de celle-ci, car ceux qui travaillent dans le tourisme sont des Wayuu qui ont des moyens, tant pour acheter des $4 \times 4$ que pour mettre en œuvre des améliorations de leurs logements

\section{Conclusion}

Le concept de l'imaginaire dialogique employé dans notre analyse nous montre comment les représentations et les attentes de chaque acteur définissent les interactions entre eux. Bien qu'au depart du parcours touristique les deux touristes de notre histoire et nous-même étions d'accord pour partager ce voyage, nous avions une base imaginaire différente. En revanche, nous pouvons qualifier les interactions entre le guide-wayuu et les touristes de complices.

Le déroulement de notre histoire nous signale le décalage existant entre le discours de l'éco- et de l'ethno-tourisme et les images dialogiques où l'image de l'autre différent - les Wayuu - reste encore associée au monde qualifié d' "arriéré ». Autrement dit, la permanence de cette image qualifiante indique et favorise l'absence de remise en question de ces touristes dans leurs appréciations du monde wayuu.

Pourrait-on penser, alors, que le discours de l'éco et de l'ethno-tourisme n'a pas encore été intégré dans les imaginaires des acteurs ? L'éco et l'ethno-tourisme resteraient comme une intention qui ne questionne nullement les vieilles représentations et deviennent une idée romantique laissant les touristes, natifs, guides, dans la croyance qu'ils sont, les uns des voyageurs " responsables », et les autres, des hôtes accueillants?

L'éco et l'ethnotourisme contribuent à l'intégration des Wayuu à l'économie de marché touristique occidentale proposée par l'État colombien au nom du développement. Mais ils y participent marginalement. Ce que les Wayuu reçoivent correspond à la représentation de ce qu' est un tourisme inconfortable et pénible, propre au "sous-développé ». On paie, donc, d'après les services donnés et dans le cadre du marché. Le prix final que paie le touriste est le prix le moins élevé de toute la liste des dépenses. En effet, les tarifs des Wayuu sont ressentis comme inacceptables par les protagonistes de notre histoire car ils voient dans leur prix la goutte qui fait déborder le vase. 


\section{Bibliographie}

ALVARADO PEREZ Margarita. " Pose y montaje en la fotografía mapuche. Retrato fotográfico, representación e identidad», pp. 13-27. Mapuche, fotografías siglos XIX y XX. Construcción y montaje de un imaginario. ALVARADO PEREZ, Margarita, MEGE ROSSO, Pedro, BAEZ ALLENDE, Christian (sous la dir. de). Santiago de Chile, Pehuén, 2001, 241 p.

- - . " Dentro y fuera del cuadro. Representación y alteridad en la fotografía de indígenas de Chile», p. 1-41. Revista chilena de antropología visual, $\mathrm{n}^{\circ} 14$ (2009).

BLANCHARD Pascal, BOËTSCH Gilles, JACOMIJN SNOEP Nanette (sous la dir. de). Exhibitions. L'Invention du sauvage, catalogue de l'exposition du Musée du Quai Branly. Paris, Actes Sud, 2011, 384 p.

CAZES Georges, COURADE Georges. «Introduction : les masques du tourisme », pp. 247-268. Tiers Monde, vol. 45, nº 178 (2004).

CHABLOZ Nadège. "Le malentendu. Les rencontres paradoxales du "tourisme solidaire" », pp. 32-47. Actes de la recherche en sciences sociales, $\mathrm{n}^{\circ} 170(2007 / 5)$.

CUNIN Élisabeth, RINAUDO Christian. «Visites guidées et marketing de la différence à Cartagena de Indias (Colombie) », pp. 137-156. Espaces et sociétés, $n^{\circ} 135(2008 / 4)$.

DOQUET Anne, LE MENESTREL Sara, "Introduction», pp. 3-13. Autrepart, ${ }^{\circ} 40(2006 / 4)$. « Tourisme culturel, réseaux et recompositions sociales ».

FINOL José Enrique. Mito y cultura guajira. Contribución a la semiótica del mito guajiro. Maracaibo, Universidad del Zulia, 2007, 300 p.

HUTIN Karen. "Quand l'intégration prépare l'exclusion : le cas des Wayuu dans la société colombienne », pp. 219-231. Alizés, n 36 (2012).

Informe socioeconómico del Departamento de La Guajira 2013. Riohacha, Cámara de Comercio de La Guajira, 2014.

Informe socioeconómico del Departamento de La Guajira 2014. Riohacha, Cámara de Comercio de La Guajira, 2015.

LOSONCZY Anne-Marie. "El criollo y el mestizo. Del sustantivo al adjetivo : categorías de apariencia y de pertenencia en la Colombia de ayer y de hoy ». Formaciones de indianidad. Articulaciones raciales, mestizaje y nación en América Latina. DE LA CADENA Marisol (sous la dir. de). Lima : Envión, 2008. pp. 261-277. Accès : http://www.cholonautas.edu.pe/2012/wp-content/ uploads/2012/04/Formaciones\%20de\%20Indianidad\%20-\%20cap\%209.pdf. Consulté le 09/11/2010.

LOSONCZY Anne-Marie, MESTURINI CAPPO Silvia. « Entre 1"Occidental" et l'Indien". Ethnographie des routes du chamanisme ayahuasquero entre Europe et Amériques », pp. 93-110. Autrepart, nº 56 (2010/4). 
MEISEL Adolfo. La Guajira y el mito de las regalías redentoras. Cartagena : Banco de la República, «Documentos de trabajo sobre economía regional Colombia », $\mathrm{n}^{\circ} 86,2007,73 \mathrm{p}$.

NAHAPETIAN Naïri, CAZES Georges. "Le tourisme enrichit les plus riches ", p. 74. Alternatives économiques, ${ }^{\circ} 235$ (2005/4).

ORSINI Giangina. Poligamia y contrabando : nociones de legalidad y legitimidad en la frontera guajira, Siglo XX. Bogotá, Universidad de los Andes-Centro de Estudios Sociales/Observatorio del Caribe Colombiano, 2007, 271 p.

PERRIN Michel. El camino de los indios muertos. Caracas, Monte Ávila, 1980, $279 \mathrm{p}$.

- - Le chemin des Indiens morts. Mythes et symboles guajiro. Paris, Payot, 1996, $263 \mathrm{p}$.

- - Les praticiens du rêve. Paris, PUF, coll. « Quadrige », 2001, 285 p.

Plan de Desarrollo del Departamento de La Guajira 2008-2011, Gobernación de La Guajira, Colombia, 2008.

POLO ACUÑA José. " Aspectos históricos de Riohacha durante el período colonial », p. 33-48. Historia Caribe, vol. 2, nº 3 (1998).

- - " "Disputas y leyes en los conflictos inter-étnicos en la península de La Guajira (Nueva Granada, 1830-1860) », pp. 69-100. Asociación Colombiana de Historia Social del Caribe, vol. 39, nº 2 (jul.-dic. 2012). Accès : http://www. revistas.unal.edu.co/index.php/achsc/article/view/37473/39827. Consulté le 15 mai 2015.

SALER Benson. «Los Wayú (Guajiro)», p. 25-146. Aborígenes de Venezuela. Etnología Contemporánea II. vol. III. Walter COPPENS, Jacques LIZOT (sous la dir. de). Caracas, Monte Ávila, 1987.

VASSEUR Marie-Thérèse. Rencontres de langues, question d'interaction. Paris, Didier, 2005, $300 \mathrm{p}$.

WINKIN Yves. Anthropologie de la communication. Paris, Seuil, 2001, 332 p.

Résumé : Grâce aux atouts naturels et culturels, l'éco-tourisme et l'ethno-tourisme s'ouvrent comme des activités économiques à La Guajira colombienne où habitent les Wayuu. Le gouvernement local prétend développer un type de tourisme auquel les Wayuu peuvent s'intégrer. Nous avons pu constater que des familles wayuu offrent leurs rancherías (logements) et proposent des activités récréatives aux touristes. Dans ce cadre, nous avons organisé un voyage en janvier 2009, avec deux touristes, avec lesquels nous étions impliquée comme guide et ethnologue. La relation triangulaire (touristes, guide natif et nous) a eu des retombées négatives et les trois acteurs ont fini le périple dans un état de déception. Quelles peuvent être les représentations de l'altérité dans un face-à-face médiatisé par le marché touristique ? Comment les places dialogiques permutent-elles et font-elles émerger des objectifs et des rôles inattendus dans les relations marchandes établies par le tourisme? Telles sont les interrogations qui ont guidé notre réflexion.

Mots-clés : éco/ethno-tourisme, Guajira colombienne, Wayuu, imaginaire dialogique, représentations. 
Resumen: Gracias a la riqueza natural de La Guajira colombiana, el eco-turismo y el etno-turismo se perfilan como una de las actividades económicas de este departamento. El gobierno local busca desarrollar un tipo de turismo en el cual los Wayúu puedan integrarse. Nosotros pudimos constatar que algunas familias wayúu ofrecen sus rancherías como hospedaje y proponen actividades lúdicas a los turistas. En este contexto, en enero de 2009, organizamos un viaje con dos turistas con los cuales estuvimos implicada como guía y como etnóloga. La relación triangular (turistas, guía nativo y nosotros) tuvo consecuencias negativas y para los tres actores el viaje fue una decepción. ¿Cuáles pueden ser las representaciones de alteridad en un cara a cara mediatizado por el mercado turístico? ¿Cómo cambian los roles y cómo emergen nuevos objetivos y roles en las relaciones mercantiles establecidas por el turismo?

Palabras clave: eco/etno-turismo, Guajira colombiana, Wayúu, imaginario dialógico, representaciones.

Biographie : Karen López Hernández a obtenu un doctorat en Anthropologie sociale et Ethnologie à l'Ecole des Hautes Etudes en Sciences Sociales (Paris). Elle est chercheuse associée au laboratoire ORACLE, de I'Université de La Réunion, ainsi qu'à l' « Observatoire de la Caraïbe colombienne » (Cartagena, Colombie). Ses thèmes de travail sont le développement, le tourisme, les comportements alimentaires, les relations entre Humains et non-Humains. Les aires géographiques concernées sont la Colombie (Wayuu) et l'Océan Indien (La Réunion, Mayotte, Comores).

Biografía: Karen López Hernández es doctora en antropología social y etnología. Obtuvo su título en I'École des Hautes Études en Sciences Sociales (París). Es miembro del laboratorio de investigación ORACLE de la Universidad de La Réunion y del Observatorio del Caribe colombiano (Cartagena, Colombia). Sus temas de investigación son el desarrollo, el turismo, los comportamientos alimentarios y las relaciones entre humanos y no-humanos, en las áreas geográficas siguientes : Colombia (Wayúu) y Océano Índico (La Reunión, Mayotte, las Comores). 